\title{
SEARCHING FOR DARK MATTER WITH \\ GRAVITATIONAL MICROLENSING: \\ A REPORT FROM THE MACHO COLLABORATION
}

\author{
C.W. STUBBS ${ }^{1,2}$ \\ ${ }^{1}$ Departments of Astronomy and Physics, \\ University of Washington, Seattle, WA 98195 \\ ${ }^{2}$ Center for Particle Astrophysics, UC Berkeley, CA 94720 \\ WITH
}

C. ALCOCK, R.A. ALlSMAN, D. ALVES, T.S. AXELROD, A. BECKER, D.P. BENNETT, K.H. COOK, K.C. FREEMAN, K. GRIEST, J. GUERN, M. LEHNER, S.L. MARSHALL, B.A. PETERSON, M.R. PRATT, P.J. QUINN, D. REISS, A.W. RODGERS, W. SUTHERLAND AND D.L. WELCH

( The MACHO Collaboration )

Center for Particle Astrophysics, Berkeley, CA 94720

Lawrence Livermore National Laboratory, Livermore, CA 94550

Mt. Stromlo and Siding Spring Observatories, Australian National University, Weston, ACT 2611, Australia

\begin{abstract}
Gravitational microlensing is the most straightforward interpretation of the stellar brightenings that have been observed by our team and other experiments. These data have provided some of the most stringent limits to date on the nature of the Galaxy's dark matter halo. The number of events seen towards the LMC indicate that our Galaxy is not surrounded by a "standard" halo of MACHOs in the mass range of $10^{-6}$ to 0.3 solar masses. The observed optical depth towards the Galactic Center is an important constraint on the distribution of mass in the plane of the Galaxy.
\end{abstract}




\section{Introduction}

The MACHO collaboration is searching for gravitational microlensing along three lines of sight, towards the LMC, the SMC and through the disk towards the Galactic center. The main objective of the project is to investigate the amount of halo dark matter that resides in lensing objects. The experiment uses a dedicated $1.3 \mathrm{~m}$ telescope at Mt. Stromlo with a dual color mosaic CCD camera system that spans 0.5 square degrees. Photometry is performed with a PSF-fitting code that is derived from DoPhot.

I want to make three main points in this talk: 1) Microlensing has in fact been detected, 2) The measured optical depth towards the LMC sets stringent limits on the nature of the Galactic dark halo, and 3) The optical depth towards the Galactic center exceeds early predictions, and understanding this will have significant implications for our understanding of the mass distribution of the Galaxy. The reader is also referred to the talks by my colleagues M. Pratt and D. Bennett for other interesting results from the MACHO project.

\section{Microlensing Has Been Detected}

When the microlensing surveys were started a few years ago, it was not at all clear that genuine events could be distinguished from background processes that would mimic microlensing. On an event-by-event basis we adopted a number of conditions to consider an excursion to be microlensing. At the time of this writing our collaboration has observed over 75 events that satisfy these criteria. The overwhelming majority are seen in the direction of the Galactic center. Other teams have also detected a significant number of candidate events, as described in other submissions to this volume.

Lack of space precludes my describing all the observed event characteristics in detail. There are two particular events that I think lend substantial credibility to the microlensing interpretation. In September of 1994 we announced (Alcock et al. 1994) the detection of a candidate event well before peak amplification. This allowed the acquisition of spectra during the event (Bennetti et al. 1995). The spectra show no evidence of variation during the course of the event. This achromaticity is a hallmark of gravitational lensing, and is strong support for the microlensing interpretation. Another event that is difficult to account for with any known intrinsic stellar variability is a case with fitted peak amplification of 18 , with detected data points at $A=15$. There is no known process other than microlensing that can give rise to such an increase in stellar flux with the observed temporal character and color-independence. Microlensing is the most straightforward interpretation. 


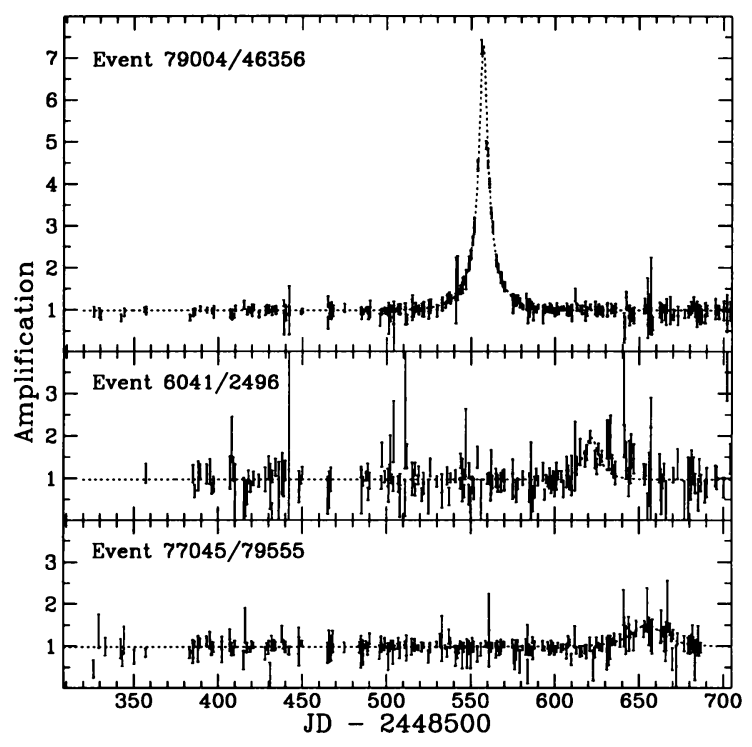

Figure 1. The three microlensing events from the MACHO project's first year LMC data. Relative flux is shown vs. time.

Any set of candidate microlensing events must then exhibit the requisite distributions in amplification and in the CM diagram. Our set of 45 candidate microlensing events from the first year's data towards the galactic bulge satisfy these requirements.

While I am certainly not claiming that all of our group's candidate events are due to microlensing, I do think that it has been established that the microlensing phenomenon is responsible for the overwhelming majority of them.

\section{Microlensing Towards the LMC, and The Galaxy's Dark Halo}

As stressed by Paczynski (1986) the optical depth towards the LMC is a sensitive way to determine the halo content of lensing objects. The LMC is the prime observing target for our experiment, and we have now completed a full analysis of the first year's data. We detected three candidate events in the first year's sample of $\sim 8.6$ million stars. The three first year LMC events that pass our selection criteria are shown in Figure 1. The experimental detection efficiency was the key ingredient that was needed in order to compare the number of detected events with the number predicted in a given halo model.

We have now completed an in-depth determination of the experiment's 


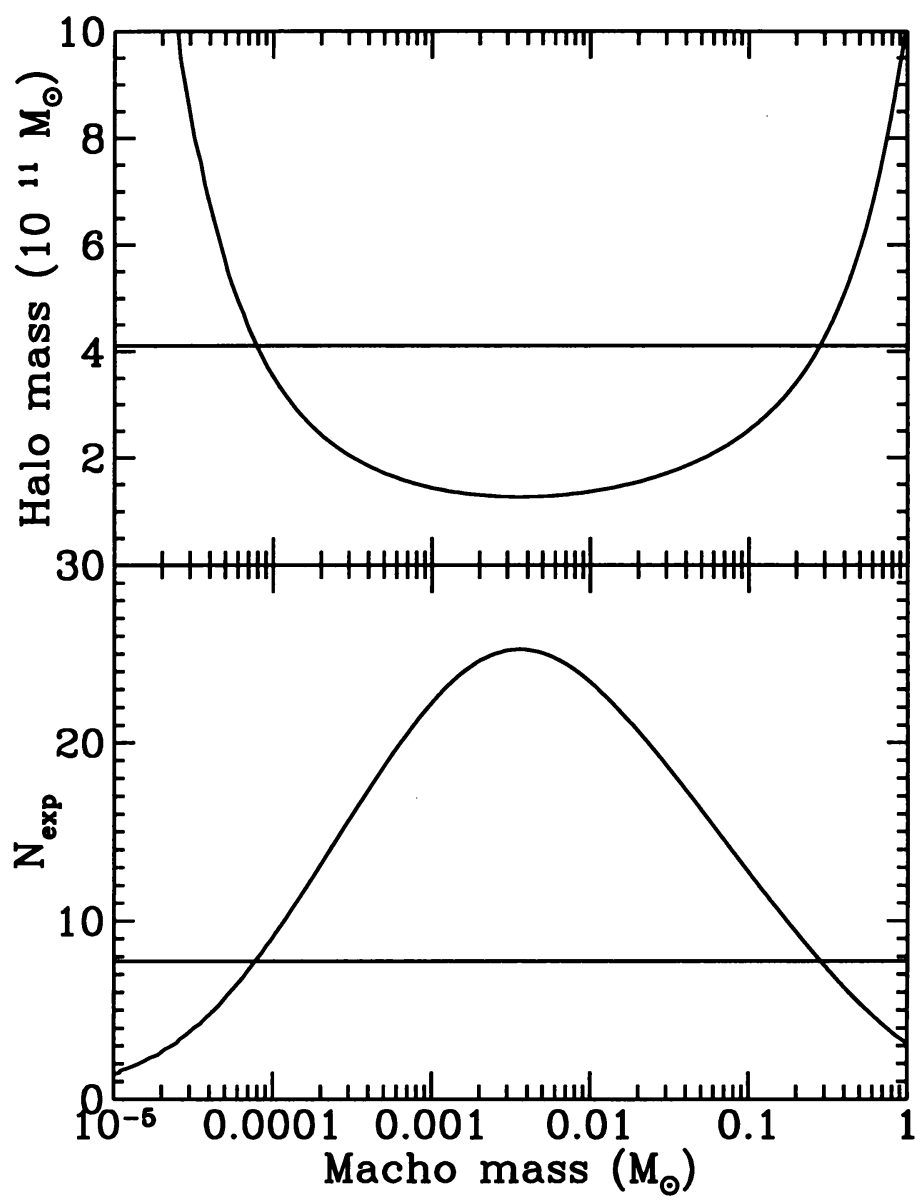

Figure 2. Constraint curves from the first year LMC data set. The $95 \%$ upper bound on the underlying event rate is 7.7 detected events, shown as the horizontal line in the lower panel. The efficiency-corrected expected number of events is the parabolic curve, assuming a $\delta$ function mass model, with mass on the $\mathrm{x}$-axis. A canonical halo of MACHOs of mass between 0.00007 and 0.3 solar masses is ruled out with high confidence. The upper panel shows the experiment's limits on total halo mass.

event detection efficiency, which is described in some detail in Alcock et al. (1995b). The fact that we operate in extremely crowded fields gives rise to two (partially offsetting) effects. First, there are more stars being monitored than one would naively expect from counting the number of "objects" that the photometry code detects. Most "objects" are in fact blends of one or more stars. This has two effects: the likelihood of detecting an event is increased since there are multiple source stars per PSF, but also there will be unamplified flux when a "blended" star is lensed, and this dilutes the 
observed amplification. We have added artificial stars to a set of frames that spans the observed seeing and sky brightness, and have determined the photometry code's response to adding flux to these objects. This set of transfer functions is used as an element in a Monte Carlo simulation that adds appropriately degraded microlensing events to a random $1 \%$ of the data set. This takes into account the actual spacing of the observations as well as blending effects and produces a matrix of detection efficiency data in amplification, event duration, and apparent magnitude of the object. Since the distribution in amplification is known a priori, we integrate out the amplification dependence, and similarly use a completeness-corrected luminosity function to integrate out the magnitude dependence. We are left with a vector of efficiency as a function of event duration that is applicable to the full data set.

The dark matter implications of our first year's LMC data have been described in two publications, Alcock et al. (1995ab). If one adopts the attitude that the detected candidate events set a upper limit on the event rate towards the LMC, the $95 \%$ upper bound on the mean detected event rate is 7.7 events. Figure 2 compares this with the efficiency-corrected expected rate for a canonical $\rho(r)=\rho_{0}\left(a^{2}+r^{2}\right)^{-1}$ halo, assuming for simplicity a delta function mass distribution in lensing objects.

A "standard" halo of lensing objects between $8 \times 10^{-5}$ and 0.3 solar masses (assuming a delta function mass distribution) is excluded with high confidence. Taken in conjunction with the null results reported in searches for shorter duration events (from the EROS CCD experiment and an unpublished analysis of the MACHO data for short events), I think it is fair to conclude that the microlensing data preclude a "standard" halo composed exclusively of objects with masses between $10^{-6}$ and 0.3 solar masses.

Naturally the next question is whether the data can accommodate other halo models. This has been treated in Alcock et al. (1995b), where we analyze a variety of scenarios, ranging from maximal disk models to the standard halo described above. It turns out that the microlensing data provide an almost model-independent constraint on the total amount of mass that resides in MACHOs, but if one wants to determine the halo fraction that this represents then our ignorance of the halo is the major limitation. It is worth stressing that the amount of dark matter support for the rotation curve at the solar circle is one of the main differences between the various contending models, and this has a direct impact on laboratory direct detection experiments for particle dark matter. I will return to this point below. 


\section{Microlensing towards the Galactic Center.}

The overwhelming majority of the events we detect are along lines of sight towards the Galactic Center. Both our team and the OGLE collaboration have estimated the bulge optical depth to be in the range of $2-4 \times 10^{-6}$, which exceeds the early predictions that assumed an axisymmetric Galactic mass distribution.

Clearly, one of the main tasks for the microlensing experiments is to map out the optical depth as a function of galactic latitude and longitude. These data must then be used in conjunction with COBE data, the rotation curve observations, high-z tracer stars, faint star counts, and other constraints to refine our knowledge of Galactic structure. Some work along these lines is beginning to appear.

Of particular interest to the dark matter community is the question of whether the maximal disk model is consistent with the data. The amount of dark matter at $\mathrm{R}=8-10 \mathrm{kpc}$ determines the event rate in both microlensing and particle dark matter direct detection experiments. This in turn depends sensitively on the extent to which the local rotation is supported by disk matter. Bulge microlensing will play in important role in establishing this, in my opinion.

The MACHO collaboration's tally of bulge events now exceeds 75 , including some exotica like lensing by binary systems (2) and one "parallax" event that shows clear effects of the earth's orbit around the sun.

\section{Conclusions}

Microlensing has been seen. The LMC data already set stringent and interesting constraints on the nature of the Galaxy's dark halo. The Galactic bulge is giving us an abundance of events that will lead to a deeper understanding of Galactic structure.

\section{References}

Alcock, C., et al., 1994, IAUC 6068

Alcock, C., et al., 1995a, PRL, 74, 2867

Alcock, C., et al., 1995b, ApJ, submitted (also astro-ph/9506113)

Benetti, S., Pasquini, L. \& West, R., 1995, A\&A, 294, L37

Paczynski, B, 1986, ApJL, 304, L1 